Eur. J. Clin. Chem. Clin. Biochem.

Vol. 31, 1993, pp. 367-374

(C) 1993 Walter de Gruyter \& Co. Berlin - New York

\title{
Calibrators and Control Samples for Bilirubinometers
}

By B. G. Blijenberg ${ }^{1}$, G. Brügmann ${ }^{2}$, W.-J. Geilenkeuser ${ }^{3}$, Regina Kusyschyn $^{2}$, G. Röhle ${ }^{3}, H$. Schlebusch $^{4}$ and Christine Schneider ${ }^{4}$

${ }^{1}$ Centraal Klinisch-Chemisch Laboratorium, Academisch Ziekenhuis Rotterdam-Dijkzigt

${ }^{2}$ Hauptlabor der Universitäts-Kinderklinik Tübingen

${ }^{3}$ Institut für Klinische Biochemie der Universität Bonn

${ }^{4}$ Abteilung für Klinische Chemie und Hämatologie, Zentrum für Geburtshilfe und Frauenheilkunde der Universität Bonn

(Received November 30, 1992/February 22, 1993)

Summary: The different matrix properties of neonatal serum and commercial control samples can lead to considerable errors in the calibration and control of bilirubinometers. These difficulties can be avoided by calibration with serum from healthy adults which is supplemented with unconjugated bilirubin. But this procedure is impracticable for most routine laboratories.

Under certain preconditions, control samples, with bilirubin concentrations determined with correctly calibrated bilirubinometers or spectrophotometers, are also suitable as calibrators. This was established by determination of the bilirubin concentration of 16 different control samples, using both the reference method and correctly calibrated bilirubinometers or spectrophotometers in three or four specialist laboratories. This was also confirmed in several interlaboratory surveys, some involving up to 72 laboratories. The results of these investigations show that a control sample should be used for the calibration of a bilirubinometer only if it meets the following preconditions:

1. There should be no significant difference between the bilirubin values determined with the reference method and with a correctly calibrated spectrophotometer or bilirubinometer.

2. The bilirubin concentration should lie in the range $230-300 \mu \mathrm{mol} / \mathrm{l}$.

The photometric response of bilirubinometers has a limited linear range, so that analytical results greater than $300 \mu \mathrm{mol} / \mathrm{l}$ must be rated as basically unreliable.

\section{Introduction}

Bilirubinometers are widely used for the determination of bilirubin in neonatal sera. These simple filter photometers use a glass capillary as the cuvette. The absorbance of undiluted serum or plasma is measured at two wavelengths, and the bilirubin concentration is computed directly.

The calibration of bilirubinometers, however, presents a serious problem. Calibration is generally performed with control sera, with nominal values stated by the supplier. The authors have already shown in previous publications $(1-4)$ that this may lead to considerable errors in the results. It was therefore suggested (5) that bilirubinometers should be calibrated with a pool of neonatal sera, using the reference method value as the assigned calibrator value. This procedure leads to a marked improvement in accuracy $(3,4)$, but it is impracticable for most laboratories, for the following reasons.

1. The collection of appropriate samples (high bilirubin concentration, no haemolysis, no phototherapy) presents many problems. 
2. Only a few laboratories are equipped to prepare a homogeneous pool and to analyse it with the reference method.

3. Extended storage, which sometimes cannot be avoided, may lead to alterations in the samples.

However, under certain conditions, correct values for neonatal sera can also be obtained by calibrating bilirubinometers with a pool of adult sera, which contains added bilirubin and which has been analysed with the reference method. This material is also suitable for testing the linear measuring range of bilirubinometers (6).

In the meantime, closer investigation of different control samples has shown that the differences between reference method values and bilirubinometer target values are large for some control sera, but small for others $(3,4,7)$. Thus, some control materials clearly have similar properties to neonatal serum when analysed by direct spectrophotometry.

The present work consists of three parts. First, the results are presented of a special survey in which the linearity and accuracy of routinely used bilirubinometers were checked. Second, reference method values and bilirubinometer target values were determined in 16 commercial control sera and their suitability as calibrators is discussed. Third, some of these samples were also used in 3 interlaboratory surveys conducted by the German Society for Clinical Chemistry in which it was determined whether the accuracy of bilirubinometer results can be improved by using such samples as calibrators.

\section{Materials and Methods}

Interlaboratory survey of the accuracy and linearity of bilirubinometers

A serum pool from 10 healthy, fasting probands (bilirubin concentration $6.0 \mu \mathrm{mol} / \mathrm{l}$ ) was equilibrated to $\mathrm{pH} 7.40$ with tonometer gas and finally supplemented with bilirubin to a concentration of $428 \mu \mathrm{mol} / \mathrm{l}$ (as determined by the reference method) (for details, see l.c. (6)).

This spiked pool was diluted in 6 steps with the starting pool, producing 6 samples with bilirubin concentrations of 377,323 , $267,215,164$ and $108 \mu \mathrm{mol} / \mathrm{l}$.

Each sample was dispensed into bilirubinometer-type capillaries, and sent by post to 54 participants in the interlaboratory survey. Tests were performed to ensure that the bilirubin concentrations of the samples did not change within 48 hours. Participants in the survey analysed the samples in the capillaries, using their apparatus as calibrated for routine analyses.

\section{Correction of results}

In each laboratory, the results for the 3 higher and 3 lower concentrations were multiplied by a correction factor. This factor was equal to the ratio of the reference method value for the intermediate sample $(267 \mu \mathrm{mol} / \mathrm{l})$ to the value obtained for that sample in the participating laboratory. Results corrected in this way represent the values that would have been expected, if all participants had used the intermediate sample as the calibrator.

Determination of reference method values and bilirubinometer target values for 16 commercially prepared control samples

Sixteen commercially prepared control specimens were investigated. In the code for each sample shown in table 1 , the letter stands for the manufacturer, whereas the numbers indicate the order of the bilirubin concentrations in the samples.

The following laboratories took part.

Laboratory 1: Centraal Klinisch-Chemisch Laboratorium, Academisch Ziekenhuis

Laboratory 2: Hauptlabor der Universitäts-Kinderklinik Tübingen

Laboratory 3: Klinisch Chemisches Laboratorium der Universitäts-Frauenklinik Bonn

Most of the reference method values were determined with the additional participation of the Institut für Klinische Chemie I der Medizinischen Hochschule Hannover.

\section{Determination of reference method values}

The method of Doumas (8) was used for the determination of reference method values. For this purpose, on two separate days the contents of 5 sample bottles were pooled and the value determined in duplicate. The volumes used in the original method were halved, and the method was otherwise unchanged. The concentration was calculated using the molar absorbance for azobilirubin of $\mathrm{A}=7550 \mathrm{~m}^{2} \cdot \mathrm{mol}^{-1}$.

Measurements were made with a spectrophotometer with a band width of $\leq 2 \mathrm{~nm}$. The accuracy of the wavelength setting was checked with holmium nitrate solution at $536.7 \mathrm{~nm}$ and $640.0 \mathrm{~nm}$. The accuracy of absorption was tested with two different cyanohaemiglobin solutions, the absorption of which had been determined at the Physikalisch-Technische Bundesanstalt (Federal Institute for Physics and Technology), Berlin.

\section{Measurements with bilirubinometers}

The following bilirubinometers were used.

Laboratory 1: Wako Bilirubin Tester (manufacturer: Wako Pure Chemical Industries Ltd., Osaka, Japan)

Laboratory 2/3: Moltronic bilirubinometer (manufacturer: Mochida Pharmaceutical Co., Tokyo, Japan)

Each apparatus was calibrated with a pool prepared from adult sera, to which bilirubin had been added to a concentration of $242 \mu \mathrm{mol} / 1$ (reference method value).

\section{Measurement of undiluted samples with spectrophotometers}

. The light path of standard cuvettes was decreased to $0.9 \mathrm{~mm}$ by using a glass insert (Hellma, No..017.000-OS). Absorbance was measured with the spectrophotometer at 455 and $575 \mathrm{~nm}$.

The supplemented serum pool described in section "Measurements with bilirubinometers" was used for calibration. 
Interlaboratory surveys with control samples as calibrators

Individual interlaboratory surveys were used to test the effect of using the same calibrators on the different bilirubinometers used by the participants. Three different versions of the survey were performed, but in each case the calibrator and the tested samples were from different sources.

\section{Version 1 (1990)}

In addition to survey samples $A$ and $B$, each participant also received a further sample with an assigned calibrator value (306 $\mu \mathrm{mol} / \mathrm{l})$. The bilirubin concentrations of samples A and B were determined first under routine conditions, then again after calibration with the calibrator provided. (This survey was performed before the determination of the data presented in table 1. The target values used are therefore absent from table 1).

\section{Version 2 (1991)}

One month after the survey, in which samples $A(e 1)$ and $B$ (e6) were measured, each participant received an additional unknown sample $C$ (b4) for a routine bilirubin determination. The values for samples $A$ and $B$ obtained by each participant were multiplied by a correction factor equal to the ratio of the reference method value for sample $C$ to the result obtained by that participant for sample $\mathrm{C}$.

The corrected results from all participants represented the survey results that would have been expected, if all participants had used the same calibrator (cf. version 1).

\section{Version 3 (1992)}

All participants received samples $\mathrm{e} 4$ and $\mathrm{b} 4$, which were designated survey sample $A$ and $B$. In analogy with version 2 , the ratio of the reference method value for sample $B$ to the parti- cipant's analytical value for sample B was calculated and used for the correction of sample A. Again, the totality of the corrected A-values represented the survey result for sample A that would have been expected, if all bilirubinometer users had measured sample $A$ after calibration of their instruments with sample B of known reference method value.

\section{Results}

Interlaboratory survey of the accuracy and linearity of bilirubinometers

The accuracy and linearity of bilirubinometers was investigated with the participation of 54 laboratories, each of which received 7 samples of different concentrations. Samples were sent to participants already dispensed into capillaries and ready for measurement. In figure $1 \mathrm{a}$, the results received are plotted against the corresponding reference method values. Figure $1 \mathrm{~b}$ shows the corresponding plot after correction of the results for calibration of each participant's bilirubinometer.

Bilirubin determinations in control samples with the reference method and by direct spectrophotometry

Results for the analysis of 16 control sera are shown in table 1. The reference method value for sample b6 was determined in November 1990, the values for the

Tab. 1. Results for the determination of target values for bilirubin in 16 control samples, using 3 analytical methods.

$\mathrm{N}=$ number of laboratories;

$\mathrm{n}=$ number of determinations;

$M_{R M}, M_{S P}, M_{B I L}=$ medians of the values from the reference method, spectrophotometry and bilirubinometry, respectively;

$R_{\max }-R_{\min }=$ difference between the highest and lowest values of $\mathrm{n}$ results.

Control Measuring principle

speci-

men

\section{Reference method}

\begin{tabular}{llll}
\hline $\mathrm{N}$ & $\mathrm{n}$ & $\dot{M}_{\mathrm{RM}}$ & $\mathrm{R}_{\max }-$ \\
& & $\mathrm{R}_{\min }$ \\
& $(\mu \mathrm{mol} / \mathrm{l})$ & $(\mu \mathrm{mol} / \mathrm{l})$
\end{tabular}

Spectrophotometer

$\mathrm{N}=3 ; \mathrm{n}=12$

\begin{tabular}{lll}
\hline$M_{S P}$ & $R_{\max }-$ & $M_{S P}-$ \\
& $R_{\min }$ & $M_{R M}$ \\
$(\mu \mathrm{mol} / \mathrm{l})$ & $(\mu \mathrm{mol} / 1)$ & $(\mu \mathrm{mol} / \mathrm{l})$
\end{tabular}

(\%)

(\%)

$(-12)$

$(+2)$

$+2$

$-1$

$-1$

$+5$

$+16$

$-10$

$+2$

$+8$

$-3$

$\begin{array}{r}-3 \\ -8 \\ \hline\end{array}$

$-17$

-17
-22

$-28$

-28
-32

$-33$

(0)

(0)

$(+2)$

(+6)

(-3)

$(+1)$

$(+3)$

$(-1)$

$(-7)$

$(-11)$

$(-11)$

$(-12)$

(-13)

$(-11)$
Bilirubinometer

$\mathrm{N}=3 ; \mathrm{n}=12$

\begin{tabular}{llll}
\hline $\mathrm{M}_{\mathrm{BIL}}$ & $\begin{array}{l}\mathrm{R}_{\max }- \\
\mathrm{R}_{\min } \\
(\mu \mathrm{mol} / \mathrm{l})\end{array}$ & $\begin{array}{l}\mathrm{M}_{\mathrm{BIL}}- \\
\mathrm{M}_{\mathrm{SP}} \\
(\mu \mathrm{mol} / \mathrm{l})\end{array}$ & $(\%)$ \\
\hline 296 & 33 & -9 & $(-3)$ \\
124 & 12 & -4 & $(-3)$ \\
219 & 12 & -3 & $(-1)$ \\
229 & 15 & 0 & $(0)$ \\
234 & 12 & -2 & $(-1)$ \\
296 & 7 & -2 & $(-1)$ \\
331 & 12 & +4 & $(+1)$ \\
309 & 15 & -4 & $(-1)$ \\
322 & 20 & +2 & $(+1)$ \\
280 & 12 & -4 & $(-1)$ \\
105 & 10 & +1 & $(+1)$ \\
146 & 9 & +4 & $(+3)$ \\
185 & 12 & +3 & $(+2)$ \\
208 & 14 & +4 & $(+2)$ \\
230 & 14 & +6 & $(+3)$ \\
266 & 16 & -2 & $(-1)$ \\
\hline
\end{tabular}

Eur. J. Clin. Chem. Clin. Biochem. / Vol. 31, 1993/ No. 6 

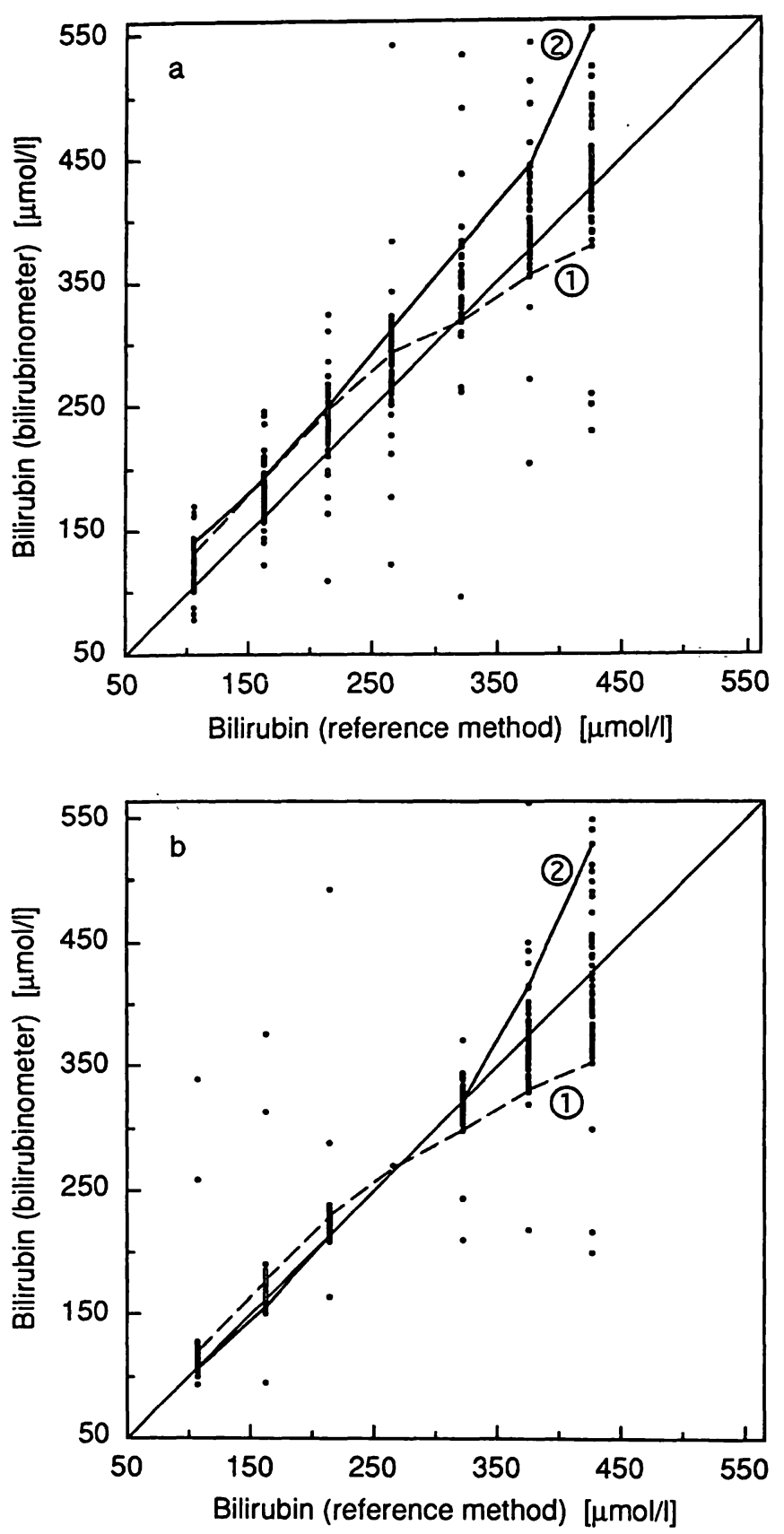

Fig. 1. Comparison of the values for bilirubin determined with bilirubinometers and with the reference method. Seven human adult pool serum samples spiked with bilirubin were analysed with 54 bilirubinometers in different laboratories.

a) Values measured in laboratories

b) Values corrected on the theoretical assumption that all the bilirubinometers had been calibrated with the intermediate sample $(267 \mu \mathrm{mol} / \mathrm{l})$.

The values of 2 individual bilirubinometers with differing characteristics are linked by lines.

other samples between March and October 1991. The results for direct spectrophotometry and for bilirubinometry were obtained in October 1991 directly after calibration of the instruments.

The median is reported for each control sample and each analytical method. The scatter of each collective of values is described here by the difference between the highest and lowest value. For direct spectrophotometric measurements, the deviation of the median of each analytical result from the median of the reference method value is given. Differences between the medians of the bilirubinometer values and the spectrophotometric values are also reported.

Interlaboratory survey results before and after calibration of the bilirubinometers

In three regular interlaboratory surveys, results were determined by normal routine procedures. In three different versions of the survey, results were also determined after correct calibration of the equipment, or the results that would have been obtained after correct calibration were calculated for the same sample material. Table 2 shows the median value and scatter for 5 different control samples in the interlaboratory survey, before and after calibration. The scatter is the difference between percentiles 16 and 84. For normally distributed results, this difference would be the range of the mean value plus and minus one standard deviation.

\section{Discussion}

Accuracy and linearity of bilirubinometers tested with bilirubin-spiked pool serum

The reliability of bilirubinometers for the determination of neonatal bilirubin has been repeatedly questioned $(1-5)$.

It has been possible to investigate neonatal sera only on a few instruments. The investigation and comparison of a relatively large number of bilirubinometers (e. g. in interlaboratory surveys) requires large quantities of sample material, so that commercially prepared control samples were used in earlier surveys. It cannot be assumed, however, that these samples are equivalent in all respects to neonatal sera.

Under these conditions, it was not possible to determine whether or to what extent unsatisfactory results were due to defects in the apparatus, inadequate quality of the sample material, errors introduced by the analyst, or some combination of these effects. A more detailed study, using adult serum supplemented with bilirubin, however, revealed a possible solution for this problem (6).

Seven different concentrations of bilirubin were prepared in adult serum, and dispensed into capillaries for the participants in the survey. Each participant received 7 capillaries, already prepared for direct 
Tab. 2. Medians and scatter ranges of the results of bilirubin determinations with bilirubinometers before and after calibration of the instruments. (For different versions of the calibration procedure, see Methods.)

\begin{tabular}{|c|c|c|c|c|c|c|c|c|c|c|c|c|c|}
\hline \multirow{4}{*}{$\begin{array}{l}\text { Inter- } \\
\text { labora- } \\
\text { tory } \\
\text { survey }\end{array}$} & \multirow{4}{*}{$\begin{array}{l}\text { Cali- } \\
\text { bra- } \\
\text { tion } \\
\text { value } \\
(\mu \mathrm{mol} / \mathrm{l})\end{array}$} & \multirow{4}{*}{ Sample } & \multirow{4}{*}{$\begin{array}{l}\text { Target } \\
\text { value } \\
\text { for } \\
\text { bili- } \\
\text { rubino- } \\
\text { meter } \\
(\mu \mathrm{mol} / \mathrm{l})\end{array}$} & \multicolumn{10}{|c|}{ Interlaboratory survey results } \\
\hline & & & & \multicolumn{5}{|c|}{ Before calibration } & \multicolumn{5}{|c|}{ After calibration } \\
\hline & & & & \multirow[t]{2}{*}{$\mathbf{n}$} & \multirow{2}{*}{$\begin{array}{l}\text { Median } \\
(\mu \mathrm{mol} / \mathrm{l})\end{array}$} & \multicolumn{2}{|c|}{ Percentile } & \multirow{2}{*}{$\begin{array}{l}\text { Differ- } \\
\text { ence } \\
84^{\text {th }}- \\
16^{\text {th }} \\
(\mu \mathrm{mol} / 1)\end{array}$} & \multirow[t]{2}{*}{ n } & \multirow{2}{*}{$\begin{array}{l}\text { Median } \\
(\mu \mathrm{mol} / \mathrm{l})\end{array}$} & \multicolumn{2}{|c|}{ Percentile } & \multirow{2}{*}{$\begin{array}{l}\begin{array}{l}\text { Differ- } \\
\text { ence }\end{array} \\
84^{\text {th }}- \\
16^{\text {th }} \\
(\mu \mathrm{mol} / \mathrm{l})\end{array}$} \\
\hline & & & & & & $\begin{array}{l}16^{\mathrm{th}} \\
(\mu \mathrm{mol} / \mathrm{l})\end{array}$ & $\begin{array}{l}84^{\mathrm{th}} \\
(\mu \mathrm{mol} / \mathrm{l})\end{array}$ & & & & $\begin{array}{l}16^{\mathrm{th}} \\
(\mu \mathrm{mol} / \mathrm{l})\end{array}$ & $\begin{array}{l}84^{\text {th }} \\
(\mu \mathrm{mol} / 1)\end{array}$ & \\
\hline 1990 & 306 & $\begin{array}{l}\text { A } \\
\text { B }\end{array}$ & $\begin{array}{l}308 \\
233\end{array}$ & $\begin{array}{l}51 \\
51\end{array}$ & $\begin{array}{l}357 \\
255\end{array}$ & $\begin{array}{l}331 \\
238\end{array}$ & $\begin{array}{l}390 \\
278\end{array}$ & $\begin{array}{l}59 \\
40\end{array}$ & $\begin{array}{l}43 \\
43\end{array}$ & $\begin{array}{l}313 \\
221\end{array}$ & $\begin{array}{l}301 \\
213\end{array}$ & $\begin{array}{l}321 \\
233\end{array}$ & $\begin{array}{l}20 \\
20\end{array}$ \\
\hline 1991 & 231 & $\begin{array}{l}\text { A (e1) } \\
\text { B (e6) }\end{array}$ & $\begin{array}{l}104 \\
267\end{array}$ & $\begin{array}{l}73 \\
73\end{array}$ & $\begin{array}{l}113 \\
289\end{array}$ & $\begin{array}{l}101 \\
265\end{array}$ & $\begin{array}{l}125 \\
314\end{array}$ & $\begin{array}{l}24 \\
49\end{array}$ & $\begin{array}{l}73 \\
73\end{array}$ & $\begin{array}{l}102 \\
262\end{array}$ & $\begin{array}{r}96 \\
248\end{array}$ & $\begin{array}{l}109 \\
274\end{array}$ & $\begin{array}{l}13 \\
26\end{array}$ \\
\hline 1992 & 231 & A (e4) & 206 & 58 & 218 & 200 & 237 & 37 & 58 & .205 & 199 & 212 & 13 \\
\hline
\end{tabular}

measurement in the bilirubinometer, and requiring no preparative procedures. This arrangement largely excluded any effects due to inadequacies of the sample material or errors by the analyst. The analytical results therefore provided a reliable indication of the analytical performance of each of the 54 bilirubinometers used for the analysis of the 7 samples. These results are summarized in figure $1 \mathrm{a}$, which shows that the 54 reported values for each of the 7 concentrations sometimes differed considerably from the reference method values.

Differences between reported values and reference method values could be due to the fact that the instruments were calibrated with different materials, whose calibration values had not been established by a common, standardized procedure.

Another important factor revealed by these results was the limited linearity of the response of each apparatus, as shown by the plot of the 7 analytical values. This is shown for two bilirubinometers in figure 1a.

Strictly speaking, Beer's law is valid only for monochromatic light and low concentrations of the analyte. As the concentration of the analyte is increased, however, its measured absorption eventually becomes measurably less than would be expected from a linear relationship between absorption and concentration. In bilirubinometers, this effect becomes increasingly evident at bilirubin concentrations above approximately $250 \mu \mathrm{mol} / 1$, as shown more or less by the performance of bilirubinometer 1 (fig. 1a). In principle, it is possible to alter the characteristics of many bilirubinometers to obtain approximate linearity, even at relatively high concentrations. This can be carried out by the service personnel, but, for technical rea- sons, apparently only to a limited extent. Thus, the performance of bilirubinometer 2 (fig. 1a) is a clear example of an overcompensated instrument setting. It should be possible for the manufacturers to achieve, at an acceptable cost, a better correlation between the measured absorption and the bilirubin concentration of a sample. For example, a small computer could be installed to correct the deviation from linearity.

However, an essential improvement in the reliability of results can be achieved even under the present conditions by providing users with the means for correctly calibrating their instruments. This is verified by figure $1 \mathrm{~b}$, which shows the results that would have been obtained for the three low and three high concentrations, if all the bilirubinometers had been calibrated using the intermediate sample and its reference method value $(267 \mu \mathrm{mol} / \mathrm{l})$. For those samples with reference method values between 108 and $323 \mu \mathrm{mol} / 1$, the scatter of the recalculated bilirubinometer values was much less than that of the original values in figure 1a. For concentrations above approximately $300 \mu \mathrm{mol} / \mathrm{l}$, however, many of the bilirubinometers no longer produced reliable results, even after precise calibration.

This may explain, at least partly, why Blijenberg et al. $(5,9)$ and other authors $(10,11)$ found a wide variation of results for values above $300 \mu \mathrm{mol} / \mathrm{l}$, even after calibration with standards based on adult serum.

It appears that a crucial improvement of results in the concentration range $100-300 \mu \mathrm{mol} / \mathrm{l}$ can be achieved by correct calibration of bilirubinometers. The question arises, however, as to which procedure is practicable for routine laboratories, which do not have a ready source of neonatal sera or supplemented adult sera for calibration purposes (see Introduction). 
These laboratories can only use commercially prepared "control samples", whose calibrator values have been established by a standardized procedure.

Bilirubin determinations by reference method and direct spectrophotometry in control samples

In an extensive investigation, 16 batches of control samples from different manufacturers were analysed by means of 3 methods: the reference method of Doumas (8), direct spectrophotometry of undiluted samples with a spectrophotometer, and with a bilirubinometer (tab. 1). The spectrometric results for each control sample were obtained within 2 days, using uniformly calibrated apparatus. Reference method values for each control sample were, however, based on ai varying number of determinations performed at different times in a different number of laboratories. The median values for the reference method were therefore based on a smaller number of analyses, but there is no evidence that this decreased their reliability.

Two aspects of table 1 are important with respect to the suitability of control samples for the calibration of bilirubinometers.

1. In both the spectrophotometric and the bilirubinometric determinations, the difference between the highest and lowest value of each collective was in many cases unexpectedly large, despite optimal calibration of the instruments. Apparently, relatively large random and systematic errors are difficult to avoid in both of these analytical methods. Much smaller differences were observed between the results obtained with the reference method.

2. In the analysis of control samples by direct photometry, there were no significant differences between the results obtained with spectrophotometers and bilirubinometers (maximal difference $3 \%$ ). In about half of the cases, however, the median values for photometry deviated markedly from the median values of the reference method. The properties of the sample material responsible for these deviations have not been identified.

Interlaboratory survey results from bilirubinometers before and after calibration with . control samples

For organizational reasons, three different procedures were used for the calibration of the bilirubinometers:
1. Forty-three of the 51 participants performed their own calibration, using a calibrator supplied to them.

2. The values were corrected on the basis of results obtained by the participant for an unknown calibrator, which the participant analysed one month after the survey.

3. One of the two survey samples served as the calibrator, in that the results were corrected on the basis of the participant's results for this sample.

After the calibration, the median values for all five samples agreed much more closely with the target values than before the calibration (for results see tab. 2). In all cases, there was a very clear improvement of interlaboratory precision, shown in the table by the differences between percentiles 84 and 16. In the second version of the calibration, this improvement might have been even more marked, if less time had elapsed between the survey and the analysis of the calibrator. It is obvious, however, that interlaboratory precision showed an especially marked improvement
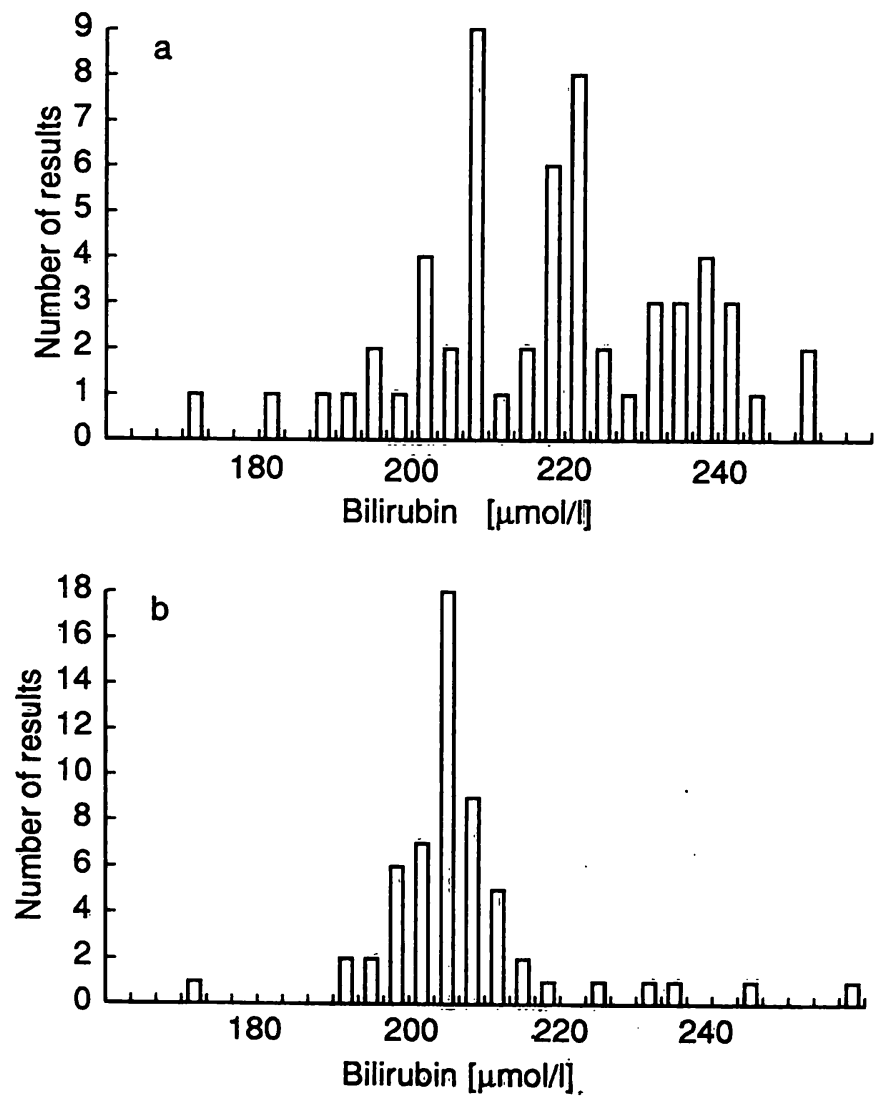

Fig. 2. Results of the determination of bilirubin in control sample $A$ of interlaboratory survey

BI $2 / 92$, using bilirubinometers.

a) Values measured in laboratories

b) Values corrected on the theoretical assumption that all bilirubinometers had been calibrated with sample B (reference method value $231 \mu \mathrm{mol} / \mathrm{l}$ ) of the interlaboratory survey (see Methods, version 3). 
whenever the concentration of the calibrator and the survey sample were close to each other, e.g. sample $A$ in the interlaboratory surveys of 1990 and 1992. (The results for sample $A$ in 1992 before and after calibration are also presented as histograms in figs. $2 a$ and $2 b$.) In both cases, the scatter of results after the calibration was only one-third of that observed before the calibration. This effect is plausibly explained by differences in the photometric performance of individual bilirubinometers (e.g. with respect to linearity), and should be taken into consideration when choosing appropriate calibrators.

\section{Conclusions}

These studies show that the reliability of bilirubinometer results can be considerably improved by standardized calibration of the apparatus. With certain preconditions, the calibration can be performed with commercially prepared control samples. These preconditions are defined and substantiated as follows.

1) The bilirubin concentration of the control material should lie between about 230 and a maximum of $300 \mu \mathrm{mol} / 1$. Since bilirubinometers do not show a uniform photometric response, they should be calibrated in the clinically most important concentration range. With modern therapeutic methods, concentrations above $300 \mu \mathrm{mol} / \mathrm{l}$ are rarely encountered in neonates. When such concentrations do occur, and especially if an exchange transfusion is under consideration, the bilirubinometer result should be checked with a different analytical method. In the great majority of cases, however, the serum bilirubin concentration is lower. On the basis of the present results, a well maintained and properly serviced bilirubinometer, correctly calibrated in the region of $250 \mu \mathrm{mol} / \mathrm{l}$, should measure concentrations in the approximate range 100-300 $\mu \mathrm{mol} / \mathrm{l}$ with sufficient reliability.

\section{References}

1. Blijenberg, B. G., Roetering, H. A. \& Leijnse, B. (1987) Reflections on the standardization of total bilirubin in neonatal serum. J. Clin. Chem. Clin. Biochem. 25, $177-$ 181.

2. Brügmann, G. (1988) Zur Kalibrierung der Bilirubinbestimmung bei Neugeborenen. J. Clin. Chem. Clin. Biochem. 26, $737-738$ (Abstract).

3. Brügmann, G. (1990) Calibration of direct reading photometers for the determination of neonatal bilirubin. J. Clin. Chem. Clin. Biochem. 28, 83-89.

4. Schlebusch, H., Liappis, N., Röhle, G. \& Schneider, Ch. (1989) Zur Kalibration von Bilirubinometern. Lab. Med. $13,364-368$.
2) The bilirubin concentration of the control material should be determined with the reference method, and the reference method value should be used as the calibration value (with certain qualifications; see below). In principle, nominal values (determined by direct photometry as described in the present work) could also be used for the calibration. It is, however, important that the value used for the calibration should have the highest possible precision and accuracy; and it has been shown that the values obtained by direct photometry, even with high performance spectrophotometers, are less precise than those obtained with the reference method. Therefore, only reference method values should be used as calibration values.

3) The reference method value of the control material and the nominal value determined by direct photometry should not differ greatly from each other (the authors recommend a maximal difference of $4 \%$ ). If the nominal value of a sample determined by direct photometry differs greatly from the reference method value, there is a high probability that any calibration performed with that sample will be wrong. Control samples showing these large differences (tab. 1) appear to be unsuitable as calibrators, but their target values provide sufficiently reliable reference points for the routine internal and external monitoring of accuracy.

Adherence to the analytical preconditions, and strict application of the standardized calibration procedures described in the present work, should produce a considerable improvement in the reliability of bilirubin determinations with bilirubinometers.

\section{Acknowledgement}

We would like to thank Prof. Dr. W.-R. Külpmann (Medizinische Hochschule Hannover) for his co-operation in the determination of reference method values.
5. Blijenberg, B. G., Roetering, H. A., De Vos, A. \& Leijnse, B. (1987) Further studies on the standardization of neonatal bilirubin. J. Clin. Chem. Clin. Biochem. 25, 737-741.

6. Schlebusch, H., Röhle, G., Schneider, Ch., Liappis, N., Geilenkeuser, W. J. \& Genz, R. (1991) Improvement of the accuracy of bilirubinometer results. Lab. Med. 15, 10-15.

7. Röhle, G., Schlebusch, H., Geilenkeuser, W. J. \& Kruse, R. (1988) External quality control in the determination of neonatal bilirubin - an approach to the improvement of results. J. Clin. Chem. Clin. Biochem. 26, $441-446$.

8. Doumas, B. T., Kwok-Cheung, P. P., Perry, B. W., Jendrzejczak, B., Mc Comb, R. B., Schaffer, R. \& Hause, L. L. (1985) Candidate reference method for determination of 
total bilirubin in serum: Development and validation. Clin. Chem. 31, 1779-1789.

9. Blijenberg, B. G. \& Leijnse, B. (1980) A survey report on the determination of total bilirubin in neonatal samples. J. Clin. Chem. Clin. Biochem. 18, 27-30.

10. St. John, A. \& Penberthy, L. A. (1979) An inter-laboratory survey of paediatric bilirubin analyses. J. Clin. Pathol. 32, $794-797$.
11. Watkinson, L. R., St. John, A. \& Penberthy, L. A. (1982) Investigation into paediatric bilirubin analyses in Australia and New Zealand. J. Clin. Pathol. 35, 52-58.

Prof. Dr. G. Röhle

Inștitut für Klinische Biochemie

der Universität Bonn

Sigmund-Freud-Straße 25

W-5300 Bonn 1

Bundesrepublik Deutschland 